\title{
Disparities in COVID-19 hospitalizations and mortality among black and Hispanic patients: cross-sectional analysis from the greater Houston metropolitan area
}

Alan P. Pan ${ }^{1}$, Osman Khan ${ }^{1}$, Jennifer R. Meeks' ${ }^{1}$, Marc L. Boom²,3, Faisal N. Masud ${ }^{3,4}$, Julia D. Andrieni ${ }^{2,3}$, Robert A. Phillips ${ }^{1,3,5}$, Yordanos M. Tiruneh ${ }^{6}$, Bita A. Kash ${ }^{1,3,7}$ and Farhaan S. Vahidy ${ }^{1,8^{*}}$ (D)

\begin{abstract}
Background: Disparate racial/ethnic burdens of the Coronavirus Disease 2019 (COVID-19) pandemic may be attributable to higher susceptibility to Severe Acute Respiratory Syndrome Coronavirus 2 (SARS-CoV-2) or to factors such as differences in hospitalization and care provision.

Methods: In our cross-sectional analysis of lab-confirmed COVID-19 cases from a tertiary, eight-hospital healthcare system across greater Houston, multivariable logistic regression models were fitted to evaluate hospitalization and mortality odds for non-Hispanic Blacks (NHBs) vs. non-Hispanic Whites (NHWs) and Hispanics vs. non-Hispanics.

Results: Between March 3rd and July 18th, 2020, 70,496 individuals were tested for SARS-CoV-2; 12,084 (17.1\%) tested positive, of whom 3536 (29.3\%) were hospitalized. Among positive cases, NHBs and Hispanics were significantly younger than NHWs and Hispanics, respectively (mean age NHBs vs. NHWs: 46.0 vs. 51.7 years; $p<0.001$ and Hispanic vs. non-Hispanic: 44.0 vs. 48.7 years; $p<0.001$ ). Despite younger age, NHBs (vs. NHWs) had a higher prevalence of diabetes (25.2\% vs. $17.6 \% ; p<0.001$ ), hypertension (47.7\% vs. $43.1 \% ; p<0.001)$, and chronic kidney disease $(5.0 \%$ vs. $3.3 \% ; p=0.001)$. Both minority groups resided in lower median income (median income [USD]; NHBs vs. NHWs: 63,489 vs. 75,793; $p<0.001$, Hispanic vs. non-Hispanic: 59,104 vs. 68,$318 ; p<0.001$ ) and higher population density areas (median population density [per square mile]; NHBs vs. NHWs: 3257 vs. 2742; $p<0.001$, Hispanic vs. non-Hispanic: 3381 vs. 2884; $p$ <0.001). In fully adjusted models, NHBs (vs. NHWs) and Hispanics (vs. non-Hispanic) had higher likelihoods of hospitalization, aOR (95\% Cl): 1.42 (1.24-1.63) and 1.61 (1.46-1.78), respectively. No differences were observed in intensive care unit (ICU) utilization or treatment parameters. Models adjusted for demographics, vital signs, laboratory parameters, hospital complications, and ICU admission vital signs demonstrated non-significantly lower likelihoods of in-hospital mortality among NHBs and Hispanic patients, aOR (95\% Cl): 0.65 (0.40-1.03) and $0.89(0.59-1.31)$, respectively.
\end{abstract}

\footnotetext{
* Correspondence: fvahidy@houstonmethodist.org

'Center for Outcomes Research, Houston Methodist, Josie Roberts

Administration Building, 7550 Greenbriar Drive, Suite 4.123, Houston, TX 77030, USA

${ }^{8}$ Houston Methodist Neurological institute, Houston Methodist, Houston, TX, USA

Full list of author information is available at the end of the article
}

(c) The Author(s). 2021 Open Access This article is licensed under a Creative Commons Attribution 4.0 International License, which permits use, sharing, adaptation, distribution and reproduction in any medium or format, as long as you give appropriate credit to the original author(s) and the source, provide a link to the Creative Commons licence, and indicate if changes were made. The images or other third party material in this article are included in the article's Creative Commons licence, unless indicated otherwise in a credit line to the material. If material is not included in the article's Creative Commons licence and your intended use is not permitted by statutory regulation or exceeds the permitted use, you will need to obtain permission directly from the copyright holder. To view a copy of this licence, visit http://creativecommons.org/licenses/by/4.0/. The Creative Commons Public Domain Dedication waiver (http://creativecommons.org/publicdomain/zero/1.0/) applies to the data made available in this article, unless otherwise stated in a credit line to the data. 
Conclusions: Our data did not demonstrate racial and ethnic differences in care provision and hospital outcomes. Higher susceptibility of racial and ethnic minorities to SARS-CoV-2 and subsequent hospitalization may be driven primarily by social determinants.

Keywords: Race, Ethnicity, Disparities, SARS-CoV-2, COVID-19

\section{Background}

The Severe Acute Respiratory Syndrome Coronavirus 2 (SARS-CoV-2) pandemic has been a global healthcare and economic catastrophe. The burden of the pandemic has been disparate, with elderly individuals and those with higher burden of cardio-metabolic diseases in the highest risk category [1, 2]. Furthermore, reports have characterized varying levels of evidence suggesting that minority populations have been disproportionately impacted by the Coronavirus Disease 2019 (COVID-19) [3, 4]. Studies from different regions and healthcare systems across the United States have broadly characterized higher susceptibility to infection and hospitalization among Black and Hispanic/Latino populations [5-9], but fewer differences with respect to mortality [10]. However, no studies, to the best of our knowledge, have systematically evaluated the associations between race/ethnicity and COVID-19 outcomes in Southeast Texas, including the greater Houston area. Such reports are particularly relevant for Black and Hispanic/Latino populations from healthcare systems that serve large, diverse populations, such as in greater Houston, where these minority racial/ethnic groups comprise of approximately 23 and $45 \%$ of the overall population, respectively [11]. Of note, in a prior analysis comparing patient characteristics between two surges in COVID-19 cases early on during the pandemic in greater Houston, the proportion of Hispanic/Latino patients from all hospitalizations increased from 25.7 to $43.3 \%$ [12]. These knowledge gaps underscore the critical need to characterize the impact of the COVID-19 pandemic on minority populations in Texas.

At the time of this reporting, the greater Houston area had begun to become a hotspot of the COVID-19 pandemic, with over 118,000 confirmed cases of COVID-19 and upwards of 2000 reported deaths [13, 14]. Given the diverse makeup of the region's population, there is value in investigating how factors related to infection, critical illness, and outcomes are associated with race and ethnicity.

Houston Methodist (HM) is one of the largest healthcare systems in the greater Houston area. Our initial report characterized the racial and ethnic disparities seen with respect to SARS-CoV-2 susceptibility, noting higher susceptibility for SARS-CoV-2 positivity among nonHispanic Black (NHB) and Hispanic populations, potentially mediated via population density and socio-economic status [15]. In this study, we evaluate racial and ethnic differences in hospitalization and in-hospital mortality among the population testing positive for SARS-CoV-2. Given our prior data, we hypothesized that Black race and Hispanic ethnicity will be independently associated with a higher hospitalization rate and in-hospital mortality in the population of COVID-19 patients across HM.

\section{Methods \\ Study setting and design}

Houston Methodist (HM) comprises a flagship tertiary care hospital and seven community and continuing care hospitals across the greater Houston area. We retrospectively analyzed data from the COVID-19 Surveillance and Outcomes Registry (CURATOR) at HM [16]. The CURA TOR is a HM Institutional Review Board (IRB) approved research registry designed to support COVID-19 research (PRO00025445). The CURATOR data are populated from electronic health records (EHR) and capture demographic, medical history, laboratory, medication, treatment, and outcomes variables for all individuals tested for SARSCoV-2 at HM. Data are continually assessed for quality and are cross validated for accuracy.

Laboratory testing for SARS-CoV-2 was performed using nucleic acid amplification tests for qualitative detection of RNA from SARS-CoV-2 isolated and purified from nasopharyngeal specimens. Diagnostic panels for real-time reverse transcription polymerase chain reaction (RT-PCR) included the Panther Fusion ${ }^{\circ}$ SARS-CoV-2 Assay and the Cepheid Xpert ${ }^{\bullet}$ Xpress SARS-CoV-2 Assay.

\section{Primary exposure and outcomes}

Predefined categories of race and ethnicity were distinctly self-reported at the time of hospital encounter. Individuals identifying primarily as Black or African American race were classified as "Black" and those identifying as Hispanic or Latino ethnicity were categorized as "Hispanic". Building on the analysis design of our prior work [15], the primary comparison categories for our study are non-Hispanic Blacks (NHBs) vs. nonHispanic Whites (NHWs), and Hispanics vs. nonHispanics. Patients who had a primary discharge diagnosis of COVID-19 (International Classification of Diseases, Tenth Revision [ICD-10]: U07.1) were classified as hospitalized with COVID-19; among those, patients who died during the episode of care were flagged for inhospital mortality. 


\section{Other covariates}

Other covariates include demographic characteristics such as age, sex, and insurance type. Patient-reported ZIP codes were used to assign population-level estimates. Median household income (inflation-adjusted to 2018 USD) was abstracted at the ZIP code tabulation area (ZCTA) level from the U.S. Census Bureau's American Community Survey 5-year estimates (2014-2018) [11]. Land area estimates were obtained from the U.S. Census Bureau's Gazetteer Files (2010) [17]. Population density (per square mile) was derived from population and land area estimates. Indices for socioeconomic status were set by designating ZIP codes (linked to ZCTA) representing the two lowest pentile groups for median income and the two highest pentile groups for population density.

Patient medical histories were queried for the presence of pre-existing conditions comprising the Charlson Comorbidity Index (CCI) [18]. Other conditions of interest include obesity, body mass index (BMI), hypertension, hyperlipidemia, asthma, smoking history, and prior vaccination history.

We extracted measurements of all systolic/diastolic blood pressure, respiratory rate, temperature, and oxygen saturation within the first $6 \mathrm{~h}$ of admission (or the first six measurements). Laboratory parameters considered in our analysis include white blood cell count, blood differential (lymphocytes), platelet count, Bnatriuretic peptide, procalcitonin, troponin, aspartate aminotransferase, alanine aminotransferase, C-reactive protein, ferritin level, D-dimer, creatinine, and venous lactate. Vital sign and laboratory measures were aggregated by taking the mean value for each patient, while excluding non-quantifiable results. Indicator flags characterizing abnormal results were derived based on commonly accepted clinical reference ranges. We excluded variables with data missingness $\geq 25 \%$ from multivariable models. Exact denominators are reported otherwise.

We extracted information on the development of hospital-associated complications (pneumonia, acute respiratory distress syndrome, bronchitis, lower respiratory tract infection, acute renal injury, acute hepatic injury, cardiomyopathy or congestive heart failure, hypoxic respiratory failure), utilization of high-acuity hospital resources such as admission to the Intensive Care Unit (ICU) and care involving use of invasive mechanical ventilation. The use of therapeutic agents such as Hydroxychloroquine, Ribavirin, Azithromycin, Lopinavir/Ritonavir, Remdesivir, Tocilizumab, Antithrombotics, Anticoagulants, and Dexamethasone were also evaluated.

\section{Statistical analysis}

Data are summarized as means (standard deviations), medians (interquartile range) or proportions based on the distribution and the functional form of the covariates. Bivariable analyses were performed to assess the associations between various socio-demographic, comorbidity and clinical factors by NHB vs. NHW race and Hispanic ethnicity. We present three explanatory logistic regression models each for the outcome of hospitalization and mortality, separately fitted for NHB (vs. NHW) race and Hispanic ethnicity (vs. nonHispanic). This approach allowed us to adjust three distinct covariate sub-sets representing varying levels of available exposure information for each outcome. For hospitalization: 1) Baseline models adjusted for age, sex, and insurance type, 2) Full socio-demographic models which included model-1 variables and added median household income and population density, and 3) Sociodemographic and comorbidity models which adds CCI, obesity, hypertension, and smoking to model-2. For inhospital mortality: 1) Baseline socio-demographic and comorbidity models including age, sex, median household income, CCI, obesity, hypertension, and smoking, 2) Hospital admission models including model-1 and vital sign and laboratory measures, and 3) Comprehensive clinical models incorporating all variables of model2 in addition to in-hospital complications, treatment course, and critical care utilization parameters. Model comparison using Akaike information criterion (AIC) was performed for each outcome.

We assessed the heterogeneity of our estimates across three strata for age $(\leq 50,51-75$, and $>75$ years $)$ and time-period of hospitalization (Phase I: Up to April 15th, 2020, Phase II: April 16th - May 31st, 2020, and Phase III: June 1st - July 18th, 2020). All data preparation and analyses were performed using $\mathrm{R}$ statistical software (version 3.6.1; The R Foundation).

\section{Results}

Between March 3rd and July 18th, 2020, a total of 70, 496 unique individuals were tested for SARS-CoV-2, of whom 12,084 (17.1\%) tested positive. The mean age across the tested population was 49.9 years and $59.9 \%$ were females. Non-Hispanic Blacks (NHB) and Hispanics comprised 19.5 and $20.7 \%$ of the tested individuals respectively. Among laboratory confirmed COVID19 cases, 3536 (29.3\%) were hospitalized, of whom 234 (6.6\%) experienced in-hospital mortality. The proportions of tested, tested-positive, hospitalized and died individuals by minority race and ethnicity across Houston Methodist are presented in Fig. 1.

\section{Baseline socio-demographic Characteristics of SARS-CoV-2 PCR-confirmed cases}

Among the total confirmed cases, 3049 (25.2\%) identified as NHB and 4372 (36.2\%) were Hispanic (Table 1). Compared to NHWs, NHBs were younger (46.0 vs. 51.7 


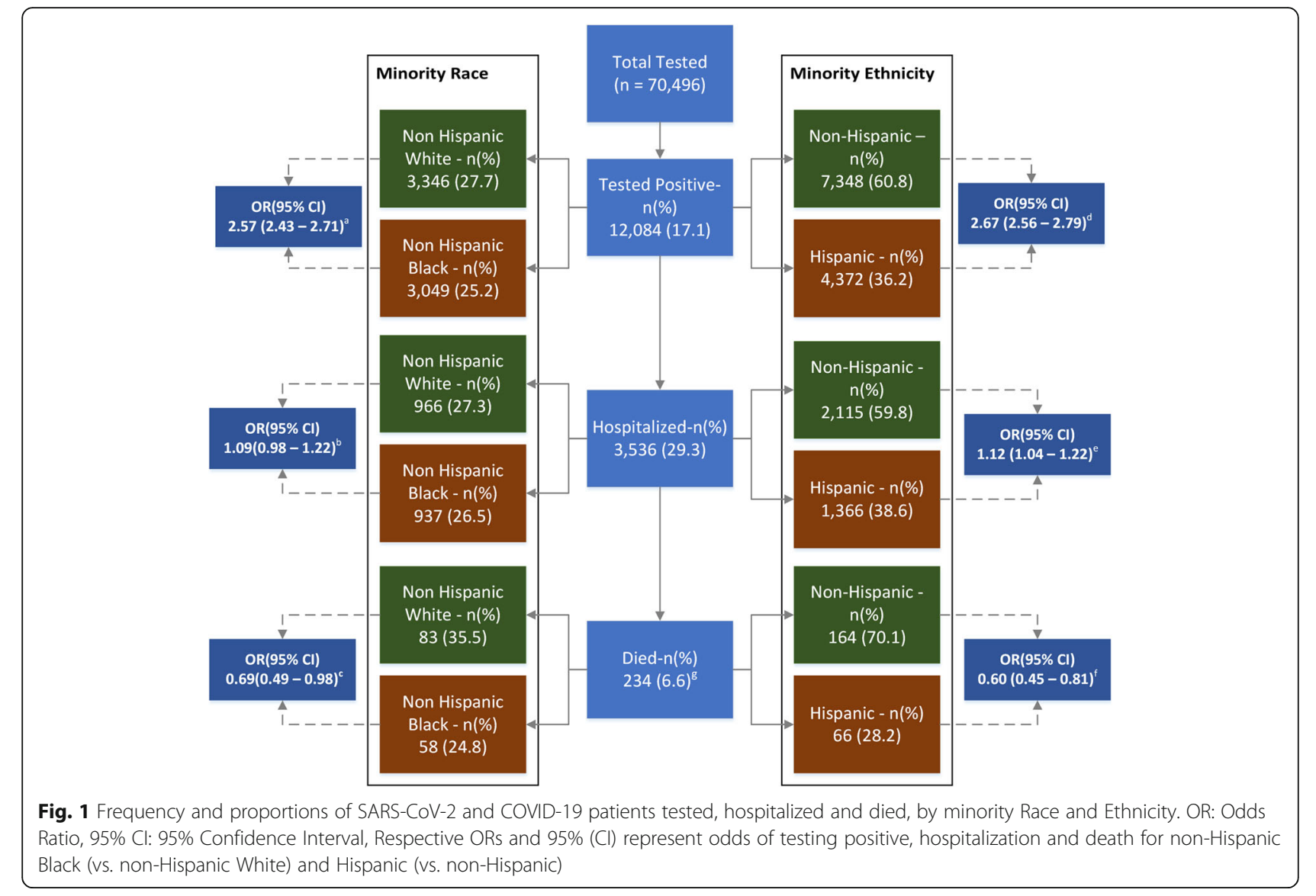

years; $p<0.001)$ with a higher proportion of females $(60.2 \%$ vs. $53.6 \% ; p<0.001)$. A greater proportion of NHBs resided in areas with lower income and higher population density. Despite younger age, NHBs (vs. NHWs) had a higher prevalence of diabetes $(25.2 \%$ vs. $17.6 \%$; $p<0.001)$, hypertension $(47.7 \%$ vs. $43.1 \%$; $p<$ $0.001)$, kidney disease $(5.0 \%$ vs. $3.3 \% ; p=0.001)$ and AIDS $(1.3 \%$ vs. $0.4 \% ; p<0.001)$. Furthermore, a greater proportion of NHBs were current or former smokers (18.9\% vs. $12.0 \% ; p<0.001)$ and had a higher mean BMI (32.2 vs. $29.8 \mathrm{~kg} / \mathrm{m}^{2} ; p<0.001$ ). Other pre-existing conditions were more prevalent among NHWs, while the overall median Charlson Comorbidity Index (CCI) was observed to be lower for NHBs (median CCI: 1 vs. 2; $p<0.001$ ). A significantly smaller proportion of NHBs were previously vaccinated for influenza $(56.9 \%$ vs. $67.7 \%$; $p<0.001)$ or pneumonia $(37.8 \%$ vs. $49.5 \%$; $p<$ $0.001)$.

Compared to non-Hispanics, Hispanics were younger (44.0 vs. 48.7 years; $p<0.001$ ) and had residence in lower income and higher population density areas. Hispanic SARS-CoV-2 positive individuals had lower prevalence of multiple pre-existing conditions, including hypertension $(31.6 \%$ vs. $43.5 \% ; p<0.001)$, myocardial infarction (MI) $(3.6 \%$ vs. $7.2 \%$; $p<0.001)$, congestive heart failure
(CHF) (4.6\% vs. 9.7\%; $p<0.001)$, peripheral vascular disease (PVD) $(4.2 \%$ vs. $7.8 \% ; p<0.001)$, chronic obstructive pulmonary disorder (COPD) (10.6\% vs. $19.2 \%$; $p<$ $0.001)$, and cancer $(3.0 \%$ vs. $6.0 \%$; $p<0.001)$. Overall comorbidity burden was also lower among Hispanics (median CCI: 1 vs. $1 ; p<0.001)$. Table 1 presents the baseline sociodemographic and comorbidity characteristics among SARS-CoV-2 PCR-confirmed cases, stratified by non-Hispanic race and Hispanic ethnicity.

\section{Racial and ethnic differences in demographic, clinical, and outcome Characteristics among hospitalized COVID-19 patients}

Among all hospitalized COVID-19 patients $(n=3536)$, NHWs and NHBs represented 27.3 and 26.5\%, respectively, whereas non-Hispanics and Hispanics represented 59.8 and $38.6 \%$, respectively. Sociodemographic and clinical differences among NHBs and NHWs and Hispanics and non-Hispanics are presented in Table 2.

NHBs were younger $(59.0$ vs. 65.0 years; $p<0.001)$ with a higher percentage of females $(54.7 \%$ vs. $45.2 \%$; $p<0.001$ ), while NHWs had a higher overall risk burden (median CCI: 5 vs. 3; $p<0.001$ ). Similarly, Hispanics were younger ( 54.2 vs. 62.1 years; $p<0.001$ ), while nonHispanics had a higher overall risk burden (median CCI: 
Table 1 Socio-Demographic and Comorbidity Characteristics of Individuals who Tested Positive $(n=12,084)$ for SARS-CoV-2, by Race and Ethnicity, at Houston Methodist through July 18th, 2020

\begin{tabular}{|c|c|c|c|c|}
\hline & $\begin{array}{l}\text { Non-Hispanic White } \\
(n=3346)\end{array}$ & $\begin{array}{l}\text { Non-Hispanic Black } \\
(n=3049)\end{array}$ & $\begin{array}{l}\text { Non-Hispanic } \\
(n=7348)\end{array}$ & $\begin{array}{l}\text { Hispanic } \\
(n=4372)\end{array}$ \\
\hline \multicolumn{5}{|l|}{ Demographics - n (\%) } \\
\hline Age - mean (SD) & $51.7(20.1)$ & $46.0(18.1)^{a}$ & $48.7(19.2)$ & $44.0(17.4)^{a}$ \\
\hline Age $\leq 50$ & $1582(47.3)$ & $1825(59.9)^{a}$ & $4008(54.5)$ & $3340(76.4)^{a}$ \\
\hline Females & $1792(53.6)$ & $1837(60.2)^{a}$ & $4164(56.7)$ & $2398(54.8)$ \\
\hline \multicolumn{5}{|l|}{ Insurance Type } \\
\hline Commercial & $1736(51.9)$ & $1454(47.7)^{a}$ & $3738(50.9)$ & $1911(43.7)^{a}$ \\
\hline Medicare & $892(26.7)$ & $567(18.6)$ & $1605(21.8)$ & $574(13.1)$ \\
\hline Medicaid & $98(2.9)$ & $227(7.4)$ & $349(4.7)$ & $380(8.7)$ \\
\hline Self-Pay & $507(15.2)$ & $729(23.9)$ & $1449(19.7)$ & $1452(33.2)$ \\
\hline Other & $113(3.4)$ & $72(2.4)$ & $207(2.8)$ & $55(1.3)$ \\
\hline Household Income (USD), Median (IQR) & $75,793(59,104-102,008)$ & $63,489(46,801-76,163)^{a}$ & $68,318(52,825-97,681)$ & $59,104(46,300-75,793)^{a}$ \\
\hline Population Density (per square mile), Median (IQR) & $2741.6(1162.5-4264.5)$ & $3256.8(2040.7-4575)^{a}$ & $2883.9(1504-4384.8)$ & $3380.9(1683.5-4384.8)^{a}$ \\
\hline \multicolumn{5}{|l|}{ Coexisting Conditions / Prior Vaccinations - n (\%) } \\
\hline CCI Total, Median (IQR) & $2(0-4)$ & $1(0-3)^{a}$ & $1(0-4)$ & $1(0-2)^{a}$ \\
\hline \multicolumn{5}{|l|}{ Charlson Comorbidities - n (\%) } \\
\hline Myocardial Infarction & $263(7.9)$ & $224(7.3)$ & $530(7.2)$ & $156(3.6)^{a}$ \\
\hline Congestive Heart Failure & $334(10.0)$ & $328(10.8)$ & $711(9.7)$ & $202(4.6)^{a}$ \\
\hline Peripheral Vascular Disease & $328(9.8)$ & $200(6.6)^{a}$ & $570(7.8)$ & $182(4.2)^{a}$ \\
\hline $\mathrm{CVA} / \mathrm{TIA}$ & $294(8.8)$ & $259(8.5)$ & $599(8.2)$ & $186(4.3)^{a}$ \\
\hline Dementia & $179(5.3)$ & $115(3.8)^{a}$ & $317(4.3)$ & $41(0.9)^{a}$ \\
\hline COPD & $712(21.3)$ & $589(19.3)$ & $1409(19.2)$ & $465(10.6)^{a}$ \\
\hline Connective Tissue Disease & $91(2.7)$ & $72(2.4)$ & 177 (2.4) & $71(1.6)^{a}$ \\
\hline Peptic Ulcer & $83(2.5)$ & $51(1.7)^{a}$ & $147(2.0)$ & $58(1.3)^{a}$ \\
\hline Liver Disease (Mild) & $238(7.1)$ & $123(4.0)^{a}$ & $410(5.6)$ & $224(5.1)$ \\
\hline Liver Disease (Moderate to Severe) & $34(1.0)$ & $12(0.4)^{a}$ & $48(0.7)$ & $34(0.8)$ \\
\hline Diabetes w/o Complications & $590(17.6)$ & $769(25.2)^{a}$ & $1541(21.0)$ & $978(22.4)$ \\
\hline Diabetes with Complications & $199(5.9)$ & $293(9.6)^{a}$ & $538(7.3)$ & $219(5.0)^{a}$ \\
\hline Hemiplegia & $40(1.2)$ & $40(1.3)$ & $87(1.2)$ & $27(0.6)^{a}$ \\
\hline CKD (Mild to Moderate) & $109(3.3)$ & $152(5.0)^{a}$ & $283(3.9)$ & $155(3.5)$ \\
\hline Solid Tumor (Localized) & $278(8.3)$ & $131(4.3)^{a}$ & $438(6.0)$ & $132(3.0)^{a}$ \\
\hline Solid Tumor (Metastatic) & $214(6.4)$ & $97(3.2)^{a}$ & $331(4.5)$ & $99(2.3)^{a}$ \\
\hline AIDS & $14(0.4)$ & $41(1.3)^{a}$ & $56(0.8)$ & $15(0.3)^{a}$ \\
\hline $\mathrm{BMl}$ - mean (SD) & $29.8(7.2)$ & $32.2(8.2)^{a}$ & $30.6(7.7)$ & $31.7(7.4)^{a}$ \\
\hline Obesity & $777(23.2)$ & $721(23.6)$ & $1614(22.0)$ & $808(18.5)^{a}$ \\
\hline Hypertension & $1441(43.1)$ & $1454(47.7)^{a}$ & 3197 (43.5) & $1380(31.6)^{a}$ \\
\hline Hyperlipidemia & $1093(32.7)$ & $826(27.1)^{a}$ & $2182(19.7)$ & $925(21.2)^{a}$ \\
\hline Asthma & $374(11.2)$ & $365(12.0)$ & $811(11.0)$ & $270(6.2)^{a}$ \\
\hline Smoking (Current/Former) & $829(24.8)$ & $577(18.9)^{a}$ & $1509(20.5)$ & $607(13.9)^{a}$ \\
\hline \multicolumn{5}{|l|}{ Prior Vaccination $^{b}$} \\
\hline Influenza & $424 / 626(67.7)$ & $370 / 650(56.9)^{a}$ & 861/1382 (62.3) & $322 / 544(59.2)^{a}$ \\
\hline Pneumonia & $527 / 1064(49.5)$ & $396 / 1047(37.8)^{a}$ & 996/2323 (42.9) & $353 / 1191(29.6)^{a}$ \\
\hline Hospitalized & 966 (28.9) & 937 (30.7) & 2115 (28.8) & $1366(31.2)^{a}$ \\
\hline
\end{tabular}

CCI Charlson Comorbidity Index, IQR Interquartile Range, CVA / TIA Cerebrovascular Accident / Transient Ischemic Attack

${ }^{a} p<0.05$

${ }^{b}$ Denominator denotes number of non-missing values

Excluding missing data:

Ethnicity $(n=364)$

Median Household Income $(n=86)$

Population Density $(n=77)$ 
4 vs. $2 ; p<0.001)$. Both minority groups were observed to reside in lower income (median income [USD]; NHBs vs. NHWs: 56,974 vs. 68,$318 ; p<0.001$, Hispanic vs. nonHispanic: 56,288 vs. 65,$805 ; p<0.001)$ and higher population density areas (median population density [per square mile]; NHBs vs. NHWs: 3257 vs. 2494; $p<0.001$, Hispanic vs. non-Hispanic: 3513 vs. $2849 ; p<0.001)$.

A greater proportion of NHBs (vs. NHWs) had mean admission temperature $\geq 38{ }^{\circ} \mathrm{C}(8.5 \%$ vs. $3.2 \% ; p<0.001)$, whereas a greater proportion of Hispanics (vs. nonHispanics) had mean oxygen saturation $<94 \%$ (19.2\% vs. $15.8 \% ; p=0.012)$ and respiratory rate $\geq 24$ breaths per $\min (25.6 \%$ vs. $18.2 \%$; $p<0.001)$. A greater proportion of NHBs had significantly elevated levels of procalcitonin (57.8\% vs. $46.7 \% ; p=0.006)$, aspartate aminotransferase (50.8\% vs. $45.4 \% ; p=0.022)$, ferritin $(9.0 \%$ vs. $4.4 \% ; p<$ $0.001)$ and creatinine $(27.4 \%$ vs. $16.1 \% ; p<0.001)$. These laboratory parameters, along with higher respiratory rates and lower oxygen saturation, were also found to be associated with mortality in our bivariable analysis for factors associated with higher in-hospital mortality (Supplemental Table 1). Among Hispanics (vs. nonHispanics), the proportion of patients with elevation of both liver enzymes (aspartate aminotransferase: 56.1\% vs. $49.8 \% ; p<0.001$ and alanine aminotransferase: $51.9 \%$ vs. $36.0 \%$; $p<0.001$ ) was higher.

Although a greater proportion of NHBs (vs. NHWs) and Hispanic (vs. non-Hispanic) patients were admitted to the ICU, these differences were not statistically significant (NHBs vs. NHWs: $31.7 \%$ vs. 29.4\%; $p=0.299$ and Hispanic vs. non-Hispanic: $31.6 \%$ vs. $31.0 \% ; p=0.711)$. Similarly, the differences in invasive mechanical ventilation utilization or days on mechanical ventilation did not reach statistical significance (Table 2).

A non-significantly higher proportion of NHBs (vs. NHWs) patients had pneumonia (65.8\% vs. $62.1 \%$; $p=$ 0.099); however, the proportion of Hispanic (vs. nonHispanic) patients with in-hospital pneumonia was significantly higher $(70.1 \%$ vs. $64.6 \% ; p=0.001)$. This finding corresponded to a higher proportion of Hispanic (vs. non-Hispanic) patients developing hypoxic respiratory failure $(43.1 \%$ vs. $36.1 \% ; p<0.001)$. Among other complications, a significantly higher proportion of NHBs (vs. NHWs) demonstrated an acute renal injury $(32.3 \%$ vs. 23.7\%; $p<0.001$ ).

We did not observe significant differences in therapeutic usage among NHBs and NHWs. A significantly smaller proportion of Hispanics (vs. non-Hispanics) received hydroxychloroquine ( $7.4 \%$ vs. $12.2 \% ; p<0.001)$, whereas the proportion of Hispanic patients receiving Remdesivir (25.3\% vs. $15.7 \%$; $p<0.001$ ) and Dexamethasone $(49.9 \%$ vs. $39.8 \% ; p<0.001)$ was significantly higher compared to non-Hispanics (Table 2).
Through July 18th, 2020, a greater proportion of NHBs vs. NHWs (30.7\% vs. $28.9 \% ; p=0.110)$ and Hispanics vs. non-Hispanics $(31.2 \%$ vs. $28.8 \% ; p=0.005)$ were hospitalized. Among discharged or deceased patients, the in-hospital mortality was significantly lower for both minority race/ethnicity groups compared with their respective counterparts (NHBs vs. NHWs: $7.6 \%$ vs. $10.7 \% ; p=0.040$ and Hispanic vs. non-Hispanic: $5.9 \%$ vs. 9.5\%; $p=0.001$ ). Similarly, lower proportional mortality for minority race/ethnicity was also observed among COVID-19 patients admitted to the ICU (NHBs vs. NHWs: $21.1 \%$ vs. $28.2 \%$; $p=0.113$ and Hispanic vs. nonHispanic: $20.4 \%$ vs. $25.9 \%$; $p=0.091$ ). The median hospital length of stay was shorter by 1 day for racial and ethnic minorities; however, the differences were not statistically significant (Table 2).

\section{Racial and ethnic differences in likelihood of hospitalization among SARS-CoV-2 positive patients}

Our unadjusted analyses demonstrated a significantly higher likelihood of SARS-CoV-2 positivity among NHB (vs. NHW) (OR, 95\% CI: 2.57, 2.45-2.71) and Hispanic (vs. non-Hispanic) (OR, 95\% CI: 2.67, 2.56-2.79) race/ ethnicity (Fig. 1). Additionally, a higher likelihood of hospitalization was observed for NHB (vs. NHW) (OR, 95\% CI: $1.09,0.98-1.22$ ) and Hispanic (vs. nonHispanic) (OR, 95\% CI: 1.12, 1.04-1.22) SARS-CoV-2 positive patients (Fig. 1). In all three multivariable model sub-sets, NHB (vs. NHW) and Hispanic (vs. nonHispanic) SARS-CoV-2 positive patients had a significantly higher likelihood of hospitalization (Table 3). Models fully adjusted for baseline socio-demographics and comorbidities (Model 3) demonstrated the best fit when assessed by comparing AIC (Table 3). In fully adjusted models, the aOR ( $95 \% \mathrm{CI}$ ) for NHBs (vs. NHWs) was 1.42 (1.24-1.63), whereas the aOR $(95 \% \mathrm{CI})$ for Hispanics (vs. non-Hispanic) was 1.61 (1.46-1.78). Other factors independently associated with higher likelihood of hospitalization are additionally reported in Table 3 . These factors also demonstrated an association with higher likelihood of hospitalization in the overall SARSCoV-2 positive population (Supplemental Table 2).

\section{Racial and ethnic differences in likelihood of death among hospitalized COVID-19 patients}

The unadjusted likelihood of in-hospital mortality was significantly lower for NHB (vs. NHW) (OR, 95\% CI: $0.69,0.49-0.98)$ and for Hispanic (vs. non-Hispanic) (OR, 95\% CI: 0.60, 0.45-0.81) COVID-19 patients (Fig. 1). Bivariable analysis of demographic and clinical factors associated with in-hospital mortality is presented in Supplemental Table 1.

Multivariable models demonstrated a non-significantly lower likelihood of in-hospital mortality among NHB 
Table 2 Clinical Characteristics of COVID-19 Hospitalized Patients $(n=3536)$, by Race and Ethnicity, at Houston Methodist through July 18th, 2020

$\begin{array}{llll}\begin{array}{l}\text { Non-Hispanic White } \\ (n=966)\end{array} & \begin{array}{l}\text { Non-Hispanic } \\ \text { Black } \\ (n=937)\end{array} & \begin{array}{l}\text { Non-Hispanic } \\ (n=2115)\end{array} & \begin{array}{l}\text { Hispanic } \\ (n=1366)\end{array}\end{array}$

\section{Demographic Characteristics and Comorbidity Index - n (\%)}

Age - mean (SD)

Female

Insurance Type

Commercial
Medicare
Medicaid
Self-Pay
Other

Household Income (USD), Median (IQR)

Population Density (per square mile), Median (IQR)

CCI Total, Median (IQR)

\section{Vital Signs at Hospital Admission - $\mathbf{n}(\%)$}

$$
\begin{aligned}
& \text { SBP }(\mathrm{mmHg})-\text { mean (SD) } \\
& \text { DBP }(\mathrm{mmHg})-\text { mean (SD) } \\
& \text { Respiratory Rate } \geq 24 \text { breath } / \mathrm{min} \\
& \text { Temperature } \geq 38^{\circ} \mathrm{C} \\
& \text { Oxygen Saturation }<94 \% \\
& \text { Laboratory Parameters - } \mathbf{n} \text { (\%) } \\
& \text { WBC count }<4000 / \mu \mathrm{l} \\
& \text { Lymphocytes }<20 \% \\
& \text { Platelet count }<150,000 / \mu \mathrm{l} \\
& \text { B-natriuretic peptide }>100 \mathrm{pg} / \mathrm{ml} \\
& \text { Procalcitonin }>0.25 \mathrm{ng} / \mathrm{ml} \\
& \text { Troponin } \geq 0.06 \mathrm{ng} / \mathrm{ml} \\
& \text { Aspartate aminotransferase }>40 \mathrm{U} / \mathrm{l} \\
& \text { Alanine aminotransferase }>40 \mathrm{U} / \mathrm{l} \\
& \text { Total Bilirubin } \geq 1.2 \mathrm{mg} / \mathrm{dl} \\
& \text { C-reactive protein }>8.2 \mathrm{ng} / \mathrm{ml} \\
& \text { Ferritin level }>3000 \mathrm{ng} / \mathrm{ml} \\
& \text { D-dimer }>0.5 \mu \mathrm{gg} / \mathrm{ml} \\
& \text { Creatinine }>1.5 \mathrm{mg} / \mathrm{dl} \\
& \text { Venous Lactate }>2.2 \mathrm{mmol} / \mathrm{l}
\end{aligned}
$$

\section{Laboratory Parameters - n (\%)}

\section{Level of Hospital Care - n (\%)}

$$
\begin{aligned}
& \text { ICU Stay }-\mathrm{n}(\%) \\
& \text { On Ventilator }-\mathrm{n}(\%) \\
& \text { Days on Ventilator, median }(\mathrm{IQR})^{\mathrm{C}}
\end{aligned}
$$

\section{Hospital Complications - $\mathbf{n}(\%)$}

Pneumonia

ARDS

Bronchitis

65.0 (17.6)

$437(45.2)$

$301(31.2)$

$478(49.5)$

$27(2.8)$

$90(9.3)$

$70(7.2)$ 829.3)

$5(2-8)$

$133.8(19.3)$
$71.5(9.3)$
$172(17.8)$
$31(3.2)$
$176(18.2)$

79/964 (8.2)

$665 / 964(69.0)$

148/964 (15.4)

296/696 (42.5)

149/319 (46.7)

206/607 (33.9)

426/938 (45.4)

322/936 (34.4)

39/861 (4.5)

$750 / 802(93.5)$

35/803 (4.4)

670/775 (86.5)

154/956 (16.1)

141/714 (19.7)

$284(29.4)$

$156(16.1)$

$6.7(2.5-13.3)$

$600(62.1)$

35 (3.6)

$12(1.2)$
$68,318(56,974-94$,

$2493.9(812.2-4081.3)$

$59.0(16.4)^{a}$

$62.1(17.3)$

$54.2(16.3)^{a}$

$513(54.7)^{a}$

1030 (48.7)

$642(47.0)$

$332(35.4)^{a}$

388 (41.4)

$50(5.3)$

$122(13.0)$

$45(4.8)$

$56,974(44,422-70,658)$

a

$3256.8(1925.8-4789.7)^{a}$

$3(1-7)^{a}$

$4(2-7)$

718 (33.9)

956 (45.2)

$82(3.9)$

$231(10.9)$

$128(6.1)$

$65,805(48,790-86$,

034)

$136.0(20.8)^{a}$

$74.1(10.6)^{a}$

$162(17.3)$

$80(8.5)^{a}$

$111(11.8)^{a}$
63/935 (6.7)

$554 / 934(59.3)^{a}$

$110 / 935(11.8)^{a}$

$213 / 646(33.0)^{a}$

$189 / 327(57.8)^{a}$

224/618 (36.2)

$467 / 919(50.8)^{a}$

317/917 (34.6)

43/865 (5.0)

725/777 (93.3)

$70 / 774(9.0)^{a}$

681/766 (88.9)

$255 / 929(27.4)^{a}$

$122 / 669(18.2)$

297 (31.7)

$162(17.3)$

$6.9(2.9-15.2)$

$617(65.8)$

$51(5.4)$

$14(1.5)$
$134.4(19.9)$

$72.6(9.9)$

$384(18.2)$

$129(6.1)$

335 (15.8)

$159 / 2111(7.5)$

$1383 / 2110(65.5)$

284/2111 (13.5)

566/1500 (37.7)

375/726 (51.7)

475/1364 (34.8)

1029/2068 (49.8)

$744 / 2064$ (36.0)

88/1930 (4.6)

1661/1775 (93.6)

114/1774 (6.4)

$1511 / 1730$ (87.3)

441/2097 (21.0)

301/1557 (19.3)

$655(31.0)$

361 (17.1)

$6.9(2.8-14.8)$

$1366(64.6)$

$109(5.2)$

$27(1.3)$ $447(32.7)^{a}$

377 (27.6)

$122(8.9)$

386 (28.3)

$34(2.5)$

$56,288(44,422-70,324)$

$3513.1(1823.2-4988.2)^{a}$

$2(1-4)^{a}$

$131.7(18.9)^{a}$

$72.2(9.3)$

$350(25.6)^{a}$

$89(6.5)$

$262(19.2)^{a}$

$74 / 1361(5.4)^{a}$

$938 / 1360(69.0)^{a}$

$145 / 1361(10.7)^{a}$

$245 / 954(25.7)^{a}$

248/526 (47.1)

$186 / 633(29.4)^{a}$

$750 / 1337(56.1)^{a}$

693/1335(51.9) ${ }^{a}$

40/1254 (3.2)

$1167 / 1199(97.3)^{a}$

65/1228 (5.3)

985/1201(82.0) ${ }^{a}$

$159 / 1346(11.8)^{a}$

191/972 (19.7)

432 (31.6)

212 (15.5)

$8.0(3.6-14.3)$

$958(70.1)^{a}$

$80(5.9)$

$16(1.2)$ 
Table 2 Clinical Characteristics of COVID-19 Hospitalized Patients $(n=3536)$, by Race and Ethnicity, at Houston Methodist through July 18th, 2020 (Continued)

\begin{tabular}{|c|c|c|c|c|}
\hline & $\begin{array}{l}\text { Non-Hispanic White } \\
(n=966)\end{array}$ & $\begin{array}{l}\text { Non-Hispanic } \\
\text { Black } \\
(n=937)\end{array}$ & $\begin{array}{l}\text { Non-Hispanic } \\
(n=2115)\end{array}$ & $\begin{array}{l}\text { Hispanic } \\
(n=1366)\end{array}$ \\
\hline Lower Respiratory Tract Infection & $12(1.2)$ & $11(1.2)$ & $24(1.1)$ & $9(0.7)$ \\
\hline Acute Renal Injury & $229(23.7)$ & $303(32.3)^{a}$ & $580(27.4)$ & $191(14.0)^{a}$ \\
\hline Acute Hepatic Injury & $16(1.7)$ & $9(1.0)$ & $28(1.3)$ & $18(1.3)$ \\
\hline Cardiomyopathy or CHF & $151(15.6)$ & $149(15.9)$ & $321(15.2)$ & $96(7.0)^{a}$ \\
\hline Hypoxic Respiratory Failure & $347(35.9)$ & $330(35.2)$ & $764(36.1)$ & $589(43.1)^{a}$ \\
\hline \multicolumn{5}{|l|}{ Therapeutics - n (\%) } \\
\hline Hydroxychloroquine & $109(11.3)$ & $123(13.1)$ & $258(12.2)$ & $101(7.4)^{a}$ \\
\hline Ribavirin & $33(3.4)$ & $29(3.1)$ & $71(3.4)$ & $31(2.3)$ \\
\hline Azithromycin & $124(12.8)$ & $150(16.0)$ & $312(14.8)$ & $208(15.2)$ \\
\hline Lopinavir/Ritonavir & $12(1.2)$ & $8(0.9)$ & $22(1.0)$ & $2(0.1)^{a}$ \\
\hline Remdesivir & $147(15.2)$ & $139(14.8)$ & $333(15.7)$ & $345(25.3)^{a}$ \\
\hline Tocilizumab & $146(15.1)$ & $149(15.9)$ & $354(16.7)$ & $320(23.4)^{a}$ \\
\hline Antithrombotic & $387(40.1)$ & $367(39.2)$ & $830(39.2)$ & $329(24.1)^{a}$ \\
\hline Anticoagulants & $874(90.5)$ & $827(88.3)$ & $1897(89.7)$ & $1246(91.2)$ \\
\hline Dexamethasone & $389(40.3)$ & $360(38.4)$ & $841(39.8)$ & $681(49.9)^{a}$ \\
\hline \multicolumn{5}{|l|}{ Outcomes - n (\%) } \\
\hline Currently Hospitalized & $182(18.8)$ & $171(18.2)$ & $391(18.5)$ & $256(18.7)$ \\
\hline Died & $84(8.7)$ & $58(6.2)^{a}$ & $164(7.8)$ & $66(4.8)^{a}$ \\
\hline Died (among discharged or died) & $84 / 784(10.7)$ & $58 / 766(7.6)^{a}$ & $164 / 1724(9.5)$ & $66 / 1110(5.9)^{a}$ \\
\hline $\begin{array}{l}\text { Died (ICU admits excl. Currently } \\
\text { hospitalized) })^{d}\end{array}$ & $60 / 213(28.2)$ & $44 / 209(21.1)^{a}$ & $124 / 478(25.9)$ & $62 / 304(20.4)^{a}$ \\
\hline Length of Stay Days - median (IQR) ${ }^{\mathrm{e}}$ & $7(3-15)$ & $6(3-12)$ & $6(3-13)$ & $5(3-10)$ \\
\hline
\end{tabular}

CCI Charlson Comorbidity Index, IQR Interquartile Range, SBP Systolic Blood Pressure, DBP Diastolic Blood Pressure, WBC White Blood Cell Count, ARDS Acute Respiratory Distress Syndrome, CHF Congestive Heart Failure

${ }^{a} p<0.05$

${ }^{b}$ Denominator denotes number of non-missing values

${ }^{c}$ Non-zero ventilator days

${ }^{d}$ Denominator denotes number of ICU admits

${ }^{e}$ LOS only for discharged or died

Excluding missing data:

Ethnicity $(n=55)$

Median Household Income $(n=34)$

Population Density $(n=30)$

(vs. NHW) and Hispanic (vs. non-Hispanic) COVID-19 patients (Table 4). Model effects were best explained (comparing AIC) when fully adjusted for sociodemographics, comorbidities, hospital course parameters, and clinical factors (Table 4; Model 3). In the fully adjusted models (Model 3), the aOR $(95 \% \mathrm{CI})$ of inhospital mortality for NHBs (vs. NHWs) was $0.65(0.40-$ 1.03) and for Hispanics (vs. non-Hispanics) was 0.89 (0.59-1.31). Advanced age, hypoxemia, lymphopenia, thrombocytopenia, elevated hepatic enzymes and creatinine, development of pulmonary and extra-pulmonary complications, and ICU admission were all independently associated with in-hospital mortality. Patients receiving Remdesivir or Dexamethasone tended to have lower likelihood of mortality.
Hospitalization and in-hospital mortality odds for race/ethnicity were examined across three strata of age and hospitalization phase. Among patients admitted across the study time periods (Phase I: Up to April 15th, 2020 [ $n=452]$, Phase II: April 16th - May 31st, 2020 [ $n=408$ ], and Phase III: June 1st - July 18th, 2020 [ $n=$ 2676]), those aged 51 to 75 years represented between 50.0 and $56.6 \%$ of hospitalizations. The evaluation of heterogeneity across the three strata of age and hospitalization phase did not yield significant interactions.

\section{Discussion}

Initial reporting from the Houston Methodist (HM) system noted increased odds of SARS-CoV-2 positivity 
Table 3 Odds Ratios for Hospitalization among SARS-CoV-2 Positive Non-Hispanic Black (vs. Non-Hispanic White) and Hispanic (vs. Non-Hispanic) Patients

\begin{tabular}{|c|c|c|c|}
\hline & $\begin{array}{l}\text { Model } 1 \\
\text { OR }(95 \% \mathrm{CI})^{\mathrm{a}} \\
(n=6395)\end{array}$ & $\begin{array}{l}\text { Model } 2 \\
\text { OR }(95 \% \mathrm{CI})^{\mathbf{a}} \\
(n=6345)\end{array}$ & $\begin{array}{l}\text { Model } 3 \\
\text { OR }(95 \% \mathrm{CI})^{\mathrm{a}} \\
(n=6344)\end{array}$ \\
\hline Model AIC & 6291.8 & 6204.9 & 5804.8 \\
\hline Non-Hispanic Black (vs. NHW) & $1.74(1.53,1.98)$ & $1.61(1.41,1.84)$ & $1.42(1.24,1.63)$ \\
\hline Age, in 5-year units & $1.30(1.27,1.34)$ & $1.31(1.28,1.34)$ & $1.18(1.15,1.22)$ \\
\hline Female (vs. Male) & $0.64(0.57,0.72)$ & $0.63(0.56,0.71)$ & $0.63(0.55,0.71)$ \\
\hline \multicolumn{4}{|l|}{ Insurance Type } \\
\hline Commercial & Reference & Reference & Reference \\
\hline Medicare & $1.66(1.39,1.98)$ & $1.58(1.32,1.89)$ & $1.14(0.94,1.39)$ \\
\hline Medicaid & $2.34(1.72,3.15)$ & $2.22(1.63,3.00)$ & $2.05(1.48,2.83)$ \\
\hline Self-Pay & $0.98(0.81,1.17)$ & $0.94(0.78,1.13)$ & $1.25(1.03,1.52)$ \\
\hline Residence: Low Income ZIP Code ${ }^{c}$ & & $1.51(1.32,1.72)$ & $1.41(1.23,1.62)$ \\
\hline Residence: Population Dense ZIP & & $0.89(0.78,1.01)$ & $0.96(0.84,1.10)$ \\
\hline Charlson Comorbidity Index Score & & & $1.15(1.12,1.19)$ \\
\hline Obesity & & & $1.50(1.29,1.74)$ \\
\hline Hypertension & & & $1.77(1.51,2.07)$ \\
\hline \multirow[t]{2}{*}{ Smoking (Current/Former) } & & & $0.94(0.80,1.10)$ \\
\hline & $\begin{array}{l}\text { Model } 1 \\
\text { OR }(95 \% \mathrm{CI})^{\mathbf{a}} \\
(n=11,720)\end{array}$ & $\begin{array}{l}\text { Model } 2 \\
\text { OR }(95 \% \mathrm{CI})^{\mathbf{a}} \\
(n=11,645)\end{array}$ & $\begin{array}{l}\text { Model } \mathbf{3} \\
\text { OR }(95 \% \mathrm{CI})^{\mathbf{a}} \\
(n=11,642)\end{array}$ \\
\hline Model AIC & 11,692 & 11,520 & 10,858 \\
\hline Hispanic (vs. Non-Hispanic) & $1.57(1.43,1.73)$ & $1.47(1.33,1.61)$ & $1.61(1.46,1.78)$ \\
\hline Age, in 5-year units & $1.30(1.28,1.33)$ & $1.31(1.29,1.33)$ & $1.21(1.18,1.23)$ \\
\hline Female (vs. Male) & $0.63(0.57,0.68)$ & $0.62(0.56,0.67)$ & $0.58(0.53,0.64)$ \\
\hline \multicolumn{4}{|l|}{ Insurance Type } \\
\hline Commercial & Reference & Reference & Reference \\
\hline Medicare & $1.70(1.48,1.95)$ & $1.63(1.42,1.87)$ & $1.23(1.06,1.42)$ \\
\hline Medicaid & $3.05(2.49,3.72)$ & $2.88(2.35,3.52)$ & $2.80(2.26,3.46)$ \\
\hline Self-Pay & $1.14(1.02,1.29)$ & $1.09(0.97,1.23)$ & $1.45(1.28,1.65)$ \\
\hline Residence: Low Income ZIPc & & $1.56(1.42,1.72)$ & $1.48(1.34,1.64)$ \\
\hline Residence: Population Dense ZIPd & & $1.00(0.91,1.10)$ & $1.07(0.97,1.18)$ \\
\hline Charlson Comorbidity Index Score & & & $1.12(1.10,1.15)$ \\
\hline Obesity & & & $1.62(1.45,1.82)$ \\
\hline Hypertension & & & $1.67(1.49,1.87)$ \\
\hline Smoking (Current/Former) & & & $0.86(0.77,0.98)$ \\
\hline
\end{tabular}

adjusted Odds Ratios and 95\% Confidence Intervals for association between individual co-variates and hospitalization among confirmed COVID-19 cases

${ }^{\mathrm{b}}$ Model Akaike information criterion (AIC)

' Low income classified as ZIP codes in the 2 lowest pentiles for median house income

${ }^{d}$ High population density classified as ZIP codes in the 2 highest pentiles for population density

among non-Hispanic Blacks (NHBs) and Hispanics [15]. Our current study expands on these findings and provides a comprehensive view of racial and ethnic disparities across varying stages in the progression of SARSCoV-2 infectivity and COVID-19 outcomes. Among PCR-confirmed SARS-CoV-2 cases, NHBs and Hispanics were more likely to be hospitalized. No differences in hospital treatment were observed; however, and adjusted in-hospital mortality was non-significantly lower for minority race/ethnicity.

Detailed reports on racial and ethnic disparities from other large healthcare systems have varied across different regions of the United States; however, our findings are similar to those reported following an early investigation from the largest healthcare system in Louisiana which observed a higher likelihood of hospitalization 
Table 4 Odds Ratios for In-Hospital Mortality among Non-Hispanic Black (vs. Non-Hispanic White) and Hispanic (vs. NonHispanic) Hospitalized COVID-19 Patients

Model AIC
Non-Hispanic Black (vs. NHW)
Demographic Factors
Age, in 5-year units
Female (vs. Male)
Residence: Low Income ZIP Code
Charlson Comorbidity Index Score
Obesity
Hypertension
Smoking (Current/Former)

\section{Vital Signs}

Respiratory Rate $\geq 24$ breath / min

Oxygen Saturation < 94\%

\section{Laboratory Parameters}

Lymphocytes $<20 \%$

Platelet count $<150,000 / \mu l$

Aspartate aminotransferase $>40 \mathrm{U} / \mathrm{I}$

Alanine aminotransferase $>40 \mathrm{U} / \mathrm{I}$

Creatinine $>1.5 \mathrm{mg} / \mathrm{dl}$

\section{Hospital Complications}

Pneumonia

ARDS

Hypoxic Respiratory Failure

Acute Renal Injury

Acute Hepatic Injury

Cardiomyopathy or CHF

\section{Therapeutics}

Remdesivir

Dexamethasone

\section{ICU Admission}

Model AIC ${ }^{\mathrm{b}}$
Hispanic (vs. Non-Hispanic)
Demographic Factors
Age, in 5-year units
Female (vs. Male)
Residence: Low Income ZIP Code
Charlson Comorbidity Index Score
Obesity
Hypertension
Smoking (Current/Former)

Model 1

OR $(95 \% \mathrm{Cl})^{\mathrm{a}}$

$(n=3454)$

1468.1

$1.12(0.80,1.54)$

$1.27(1.20,1.35)$

$0.78(0.58,1.04)$

$0.95(0.72,1.28)$

$1.11(1.06,1.16)$

$1.30(0.94,1.78)$

$1.38(0.88,2.21)$

$1.16(0.84,1.60)$

$\begin{array}{ll}\text { Model 2 } & \text { Model 3 } \\ \text { OR }(\mathbf{9 5} \% \mathrm{Cl})^{\mathbf{a}} & \text { OR }(\mathbf{9 5} \% \mathrm{Cl})^{\mathbf{a}} \\ (n=1835) & (n=1835) \\ 796.86 & 682.03\end{array}$

$0.72(0.47,1.09)$

$0.65(0.40,1.03)$

$1.26(1.16,1.38)$
$1.13(0.76,1.69)$
$0.95(0.64,1.40)$
$1.09(1.03,1.16)$
$1.18(0.77,1.81)$
$0.91(0.48,1.85)$
$1.04(0.69,1.57)$

$1.31(1.20,1.45)$

$1.00(0.64,1.56)$

$0.95(0.62,1.47)$

$1.03(0.96,1.10)$

$1.04(0.65,1.67)$

$0.81(0.39,1.77)$

$1.11(0.71,1.74)$

$1.80(1.16,2.75)$

$1.29(0.79,2.08)$

$1.94(1.21,3.09)$

$1.75(1.03,2.93)$

$3.05(1.77,5.57)$

$2.30(1.27,4.41)$

$2.02(1.26,3.20)$

$1.93(1.14,3.25)$

$2.32(1.46,3.72)$

$2.18(1.31,3.66)$

$1.04(0.66,1.66)$

$0.76(0.45,1.28)$

$2.99(1.97,4.55)$

$2.20(1.37,3.55)$

$1.04(0.58,1.90)$

$5.53(2.60,11.91)$

$3.68(2.15,6.45)$

$1.88(1.17,3.01)$

$1.23(0.40,3.61)$

$1.10(0.67,1.77)$

$0.56(0.26,1.12)$

$0.56(0.26,1.12)$

$0.64(0.40,1.00)$

$3.43(2.08,5.72)$

Model 2

OR $(95 \% \mathrm{Cl})^{\mathrm{a}}$

$(n=3369)$

Model 3

OR $(95 \% \mathrm{Cl})^{\mathrm{a}}$

$(n=3369)$

1261.7

$0.95(0.67,1.34)$

1012.8

$0.89(0.59,1.31)$

$1.29(1.21,1.39)$
$1.09(0.80,1.49)$
$0.87(0.64,1.18)$
$1.09(1.04,1.14)$
$1.34(0.95,1.88)$
$1.09(0.68,1.78)$
$1.12(0.79,1.58)$

$1.35(1.25,1.46)$

$0.93(0.65,1.32)$

$0.88(0.63,1.25)$

$1.02(0.96,1.08)$

$1.08(0.73,1.58)$

$0.88(0.52,1.54)$

$1.25(0.85,1.84)$ 
Table 4 Odds Ratios for In-Hospital Mortality among Non-Hispanic Black (vs. Non-Hispanic White) and Hispanic (vs. NonHispanic) Hospitalized COVID-19 Patients (Continued)

\begin{tabular}{|c|c|c|}
\hline \multicolumn{3}{|l|}{ Vital Signs } \\
\hline Respiratory Rate $\geq 24$ breath / min & $1.44(1.02,2.01)$ & $1.13(0.77,1.66)$ \\
\hline Oxygen Saturation < 94\% & $2.33(1.64,3.31)$ & $1.90(1.27,2.84)$ \\
\hline \multicolumn{3}{|l|}{ Laboratory Parameters } \\
\hline Lymphocytes < 20\% & $3.45(2.14,5.86)$ & $2.31(1.36,4.08)$ \\
\hline Platelet count $<150,000 / \mu \mathrm{l}$ & $1.99(1.37,2.86)$ & $1.60(1.03,2.45)$ \\
\hline Aspartate aminotransferase $>40 \mathrm{U} / \mathrm{I}$ & $2.88(1.96,4.27)$ & $2.47(1.61,3.82)$ \\
\hline Alanine aminotransferase $>40 \mathrm{U} / \mathrm{l}$ & $1.14(0.80,1.64)$ & $0.74(0.49,1.13)$ \\
\hline Creatinine $>1.5 \mathrm{mg} / \mathrm{dl}$ & $3.04(2.18,4.23)$ & $1.98(1.35,2.89)$ \\
\hline \multicolumn{3}{|l|}{ Hospital Complications } \\
\hline Pneumonia & & $1.00(0.60,1.67)$ \\
\hline ARDS & & $7.02(3.82,13.12)$ \\
\hline Hypoxic Respiratory Failure & & $3.67(2.27,6.07)$ \\
\hline Acute Renal Injury & & $2.04(1.40,2.97)$ \\
\hline Acute Hepatic Injury & & $3.31(1.46,7.58)$ \\
\hline Cardiomyopathy or CHF & & $1.21(0.80,1.80)$ \\
\hline \multicolumn{3}{|l|}{ Therapeutics } \\
\hline Remdesivir & & $0.57(0.34,0.95)$ \\
\hline Dexamethasone & & $0.73(0.51,1.06)$ \\
\hline ICU Admission & & $4.55(2.97,7.08)$ \\
\hline
\end{tabular}

AIC Akaike information criterion, ARDS Acute Respiratory Distress Syndrome, CHF Congestive Heart Failure

${ }^{a}$ Adjusted Odds Ratios and 95\% Confidence Intervals for association between individual co-variates and in-hospital mortality among confirmed COVID-19 cases

${ }^{\mathrm{b}}$ Model Akaike information criterion (AIC)

c Low income classified as ZIP codes in the 2 lowest pentiles for median house income

among NHBs but no differences with respect to inhospital mortality [19]. Similarly, analyses from another single medical center in New York City reported no differences in hospital admission or in-hospital mortality for NHBs; however, Hispanics experienced increased likelihoods of both hospitalization and in-hospital mortality [20]. Overall, higher susceptibility to infection and hospitalization has been broadly observed for minority groups - particularly Blacks [5-8] and Hispanics [9] by a range of other studies, with fewer differences found with respect to mortality. Of note, although in our data, specific disease conditions (e.g., diabetes) were reportedly more prevalent among hospitalized NHBs (vs. NHW), overall comorbidity burden was not higher for either minority race/ethnicity group. In addition, the average age of SARS-CoV-2 infections and COVID-19 hospitalizations in our cohort was observed to be lower for NHBs (vs. NHW) and Hispanics (vs. non-Hispanic) which may have contributed relative protection for these patients. Lastly, we note that standardized and intensive care across our hospitals may have narrowed differences in severe disease outcomes. These considerations may partly explain why in-hospital mortality did not differ. It is therefore likely that the population-level burden of SARS-CoV-2 infection and COVID-19-related morbidity and mortality is driven primarily by a greater susceptibility to infection, rather than disparities related to care provision. It is critical to identify specific population-level targets for mitigating the disproportionate impact of the SARS-CoV-2 burden and similar health catastrophes among communities of color.

Interpretation of racial and ethnic disparities should also consider the distinct populations for which epidemiological analyses are being conducted. Whereas our hospital system data demonstrate a greater than twofold likelihood of SARS-CoV-2 infection among both racial and ethnic minority groups, publicly available data indicate that, in Houston, the population ratio of Black-toWhite race is 0.91 but among the SARS-CoV-2 positive cases this ratio is 0.84 (demonstrating proportionately lower infection rates among Blacks) (Supplemental Table 3). Conversely, the population ratio of Hispanic-to-nonHispanic ethnicity is 0.81 but this ratio in the SARS$\mathrm{CoV}-2$ positive population is 0.85 . Given this contrast between our findings and publicly reported data, it is important to note that the ratios for infection rates presented above reflect a snapshot of case prevalence. Our analyses do attempt to account for this when comparing the likelihoods of infection, hospitalization, or inhospital mortality among our base cohorts. Furthermore, 
the differences may be explained, in part, by testing characteristics across a healthcare system; our data reflect characteristics of individuals who sought out pointof-care testing but do not capture the full scope of testing that is occurring at public or community-based sites across greater Houston. Presumably, we might expect differences in the location of preference for testing, based on an individual's perception of need or convenience. In contrast to what we observed in Houston, the population Black-to-White ratio in New Orleans is 1.95, but the SARS-CoV-2 infection and death Black-toWhite ratios are 3.20 and 3.25 , respectively. Similarly, higher Black-to-White ratios for SARS-CoV-2 infection, hospitalization and mortality (compared with the population Black-to-White ratio) were observed for New York City and Chicago. Supplemental Table 3 presents available case and region demographics for a selection of major U.S. metropolitan areas. However, several gaps remain in reporting of race and ethnicity-based metrics.

Limitations of our study include use of data from a single healthcare system across the greater Houston area. Although HM serves patient populations in communities located beyond the metropolitan area, hospital data may not be representative of a generalized population. Moreover, our work is driven by analysis of healthcare utilization data; consequently, it is likely that differences in care utilization would influence the patient population represented in our data. Whereas NHBs and Hispanics make up an approximate 23 and $45 \%$ of the greater Houston area population [11], they comprised only 19.5 and $20.7 \%$ of our testing cohort. Given these differences, disproportionate testing characteristics may have biased our data. The findings from our study need to be replicated across large and diverse healthcare systems and evaluated in light of specific demographic contexts in the region. Additionally, given the observational nature of our study, all estimates are associative and do not imply causation. In particular, we did not perform adjusted analyses for factors associated with hospitalization or mortality, as that was not the focus of this study. Any association observed in our bivariable analysis of factors related to morality or hospitalization would warrant separate validation analyses to be of interpretational value. Although our EHR captures a comprehensive array of information reported across encounters at our hospital system, a complete account of individual medical histories is not available. Median household income and population density were included as approximations of socioeconomic and social distancing challenges; however, the true effects are undoubtedly more complex and multifactorial in nature $[21,22]$. Future analyses should employ additional population measures to account for social determinants of health.

\section{Conclusion}

The need to identify the root causes of the disparate burden of the SARS-CoV-2 pandemic among communities of color as precisely as possible should lead healthcare officials and institutions to focus the public health messaging on appropriate targets. Such efforts would be particularly valuable for mitigating disparities at the onset of future public health crises. Our data demonstrate that, despite a higher likelihood of infection and hospitalization among NHB and Hispanic populations, there were no subsequent differences in hospital treatment or in-hospital mortality. These findings support the hypothesis that the overall higher burden of the COVID-19 pandemic among racial and ethnic minorities may be driven by higher susceptibility to contracting the SARS-CoV-2. Such susceptibilities are most likely mediated by adverse social determinants of health, which puts these minority populations at risk of infection in the community setting.

\section{Abbreviations}

aOR: Adjusted odds ratio; CVA / TIA: Cerebrovascular accident / transient ischemic attack; CCl: Charlson Comorbidity Index; COPD: Chronic obstructive pulmonary disorder; Cl: Confidence interval; CHF: Congestive heart failure; COVID-19: Coronavirus Disease 2019; CURATOR: COVID-19 Surveillance and Outcomes Registry; HM: Houston Methodist; ICU: Intensive Care Unit; MI: Myocardial infarction; NHB: Non-Hispanic Black; NHW: Non-Hispanic White; OR: Odds ratio; PVD: Peripheral vascular disease; RT-PCR: Real-time reverse transcription polymerase chain reaction; SARS-CoV-2: Severe Acute Respiratory Syndrome Coronavirus 2; ZCTA: ZIP Code Tabulation Area

\section{Supplementary Information}

The online version contains supplementary material available at https://doi. org/10.1186/s12889-021-11431-2.

Additional file 1: Supplemental Table 1. Socio-Demographic, Comorbidity, and Clinical Factors Associated with In-Hospital Mortality among Hospitalized COVID-19 Patients. Supplemental Table 2. Baseline SocioDemographic and Comorbidity Factors Associated with Hospitalization among SARS-CoV-2 Positive Individuals. Supplemental Table 3. Differences in COVID-19 Case Demographics for Select U.S. Metropolitan Areas.

\section{Acknowledgements}

None.

\section{Authors' contributions}

FV was responsible for the study conception and design. Data acquisition and management was overseen by AP, OK and JM. Statistical analysis was performed by AP. Interpretation of results and initial drafting of the manuscript were completed by AP and FV. MB, FM, JA, RP, YT, and BK made critical revisions to the manuscript draft. All authors approved the final version for submission.

\section{Funding}

No funding was received for conducting this study.

Availability of data and materials

Data cannot be shared publicly because of patient confidentiality concerns. Data are available from the Houston Methodist Institutional Data Access / Ethics Committee (contact via fvahidy@houstonmethodist.org) for researchers who meet the criteria for access to confidential data. 


\section{Declarations}

\section{Ethics approval and consent to participate}

The study protocol and ethics approval (PRO00025445) was granted by the Houston Methodist Institutional Review Board. The study protocol was approved to operate under a waiver of informed consent as outlined in 45 CFR 46.116(f)(iii)

\section{Consent for publication}

Not applicable.

\section{Competing interests}

The authors declare that they have no competing interests.

\section{Author details}

'Center for Outcomes Research, Houston Methodist, Josie Roberts Administration Building, 7550 Greenbriar Drive, Suite 4.123, Houston, TX 77030, USA. ${ }^{2}$ Department of Clinical Medicine, Houston Methodist, Houston, TX, USA. ${ }^{3}$ Weill Cornell Medical College, New York, NY, USA. ${ }^{4}$ Department of Anesthesiology and Critical Care, Houston Methodist, Houston, TX, USA. ${ }^{5}$ Department of Cardiology, Houston Methodist, Houston, TX, USA. ${ }^{6}$ University of Texas Health Science Center at Tyler, Tyler, TX, USA. ${ }^{7}$ Texas A\&M University School of Public Health, College Station, TX, USA. ${ }^{8}$ Houston Methodist Neurological institute, Houston Methodist, Houston, TX, USA.

\section{Received: 28 October 2020 Accepted: 30 June 2021}

Published online: 06 July 2021

\section{References}

1. Garg S. Hospitalization Rates and Characteristics of Patients Hospitalized with Laboratory-Confirmed Coronavirus Disease 2019 - COVID-NET, 14 States, March 1-30, 2020. MMWR Morb Mortal Wkly Rep. 2020;69 [cited 2020 Jul 2] Available from: https://www.cdc.gov/mmwr/volumes/69/wr/ mm6915e3.htm

2. Stokes EK. Coronavirus Disease 2019 Case Surveillance - United States, January 22-May 30, 2020. MMWR Morb Mortal Wkly Rep. 2020;69 [cited 2020 Jul 2] Available from: https://www.cdc.gov/mmwr/volumes/69/wr/ mm6924e2.htm

3. Centers for Disease Control and Prevention. Health Equity Considerations and Racial and Ethnic Minority Groups. Coronavirus Disease 2019 (COVID19). 2020. Available from: https://www.cdc.gov/coronavirus/2019-ncov/ need-extra-precautions/racial-ethnic-minorities.html.

4. Artiga S, Garfield R, Orgera K. Communities of color at higher risk for health and economic challenges due to COVID-19. Kaiser Family Foundation 2020. Available from: https://www.kff.org/coronavirus-covid-19/issue-brief/ communities-of-color-at-higher-risk-for-health-and-economic-challengesdue-to-covid-19.

5. Azar KMJ, Shen Z, Romanelli RJ, Lockhart SH, Smits K, Robinson S, et al. Disparities In Outcomes Among COVID-19 Patients In A Large Health Care System In California. Health Affairs. 2020 [cited 2020 May 26] Available from. https://doi.org/10.1377/hlthaff.2020.00598.

6. Gold JAW. Characteristics and Clinical Outcomes of Adult Patients Hospitalized with COVID-19 — Georgia, March 2020. MMWR Morb Mortal Wkly Rep. 2020;69 [cited 2020 Jul 2] Available from: https://www.cdc.gov/ mmwr/volumes/69/wr/mm6918e1.htm.

7. Killerby ME. Characteristics Associated with Hospitalization Among Patients with COVID-19 - Metropolitan Atlanta, Georgia, March-April 2020. MMWR Morb Mortal Wkly Rep. 2020;69 [cited 2020 Jul 2] Available from: https:// www.cdc.gov/mmwr/volumes/69/wr/mm6925e1.htm.

8. Suleyman G, Fadel RA, Malette KM, Hammond C, Abdulla H, Entz A, et al. Clinical Characteristics and Morbidity Associated With Coronavirus Disease 2019 in a Series of Patients in Metropolitan Detroit. JAMA Netw Open. 2020; 3(6):e2012270 [cited 2020 Jul 1] Available from: https://jamanetwork.com/ journals/jamanetworkopen/fullarticle/2767216.

9. Martinez DA, Hinson JS, Klein EY, Irvin NA, Saheed M, Page KR, et al. SARSCoV-2 Positivity Rate for Latinos in the Baltimore-Washington, DC Region. JAMA. 2020; [cited 2020 Jun 25]; Available from: https://jamanetwork.com/ journals/jama/fullarticle/2767632.

10. Asch DA, Islam MN, Sheils NE, Chen Y, Doshi JA, Buresh J, et al. Patient and Hospital Factors Associated With Differences in Mortality Rates Among Black and White US Medicare Beneficiaries Hospitalized With COVID-19 Infection.
JAMA Netw Open. 2021;4(6):e2112842 [cited 2021 Jun 17] Available from: https://jamanetwork.com/journals/jamanetworkopen/fullarticle/2781182.

11. United States Census Bureau. American community survey (ACS). 2018. Available from: https://www.census.gov/programs-surveys/acs.

12. Vahidy FS, Drews AL, Masud FN, Schwartz RL, Askary B"B", Boom ML, et al. Characteristics and Outcomes of COVID-19 Patients During Initial Peak and Resurgence in the Houston Metropolitan Area. JAMA. 2020;324(10):998 [cited 2021 Feb 8] Available from: https://jamanetwork.com/journals/jama/ fullarticle/2769610.

13. Texas Health and Human Services. Coronavirus disease 2019 (COVID-19). 2020. Available from: https://www.dshs.texas.gov/coronavirus.

14. Texas Medical Center. Coronavirus (COVID-19) Updates. 2020. Available from: https://www.tmc.edu/coronavirus-updates./

15. Vahidy FS, Nicolas JC, Meeks JR, Khan O, Pan A, Jones SL, et al. Racial and ethnic disparities in SARS-CoV-2 pandemic: analysis of a COVID-19 observational registry for a diverse US metropolitan population. BMJ Open. 2020;10(8):e039849. https://doi.org/10.1136/bmjopen-2020-039849.

16. Vahidy F, Jones SL, Tano ME, Nicolas JC, Khan OA, Meeks JR, et al. Rapid Response to Drive COVID-19 Research in a Learning Health Care System: Rationale and Design of the Houston Methodist COVID-19 Surveillance and Outcomes Registry (CURATOR). JMIR Med Inform. 2021 Feb 23 [cited 2021 Feb 13];9(2):e26773. Available from: http://medinform.jmir.org/2021/2/e2 $6773 /$.

17. United States Census Bureau. Gazetteer Files. Available from: https://www. census.gov/geographies/reference-files/time-series/geo/gazetteer-files.html.

18. Charlson ME, Pompei $\mathrm{P}$, Ales $\mathrm{KL}$, MacKenzie CR. A new method of classifying prognostic comorbidity in longitudinal studies: Development and validation. J Chronic Dis. 1987;40(5):373-83 [cited 2020 Jul 1] Available from: https:// linkinghub.elsevier.com/retrieve/pii/0021968187901718.

19. Price-Haywood EG, Burton J, Fort D, Seoane L. Hospitalization and Mortality among Black Patients and White Patients with Covid-19. N Engl J Med. 2020; [cited 2020 May 31] Available from: http://www.nejm.org/doi/10.1056/ NEJMsa2011686.

20. Petrilli CM, Jones SA, Yang J, Rajagopalan H, O'Donnell L, Chernyak Y, et al. Factors associated with hospital admission and critical illness among 5279 people with coronavirus disease 2019 in New York City: prospective cohort study. BMJ. 2020;369 [cited 2020 May 26] Available from: https://www.bmj. com/content/369/bmj.m1966.

21. Braveman P, Gottlieb L. The Social Determinants of Health: It's Time to Consider the Causes of the Causes. Public Health Rep. 2014;129(1_suppl2): 19-31 [cited 2020 Jul 26] Available from: http://journals.sagepub.com/doi/1 $0.1177 / 003335491412915206$

22. The Relative Contribution of Multiple Determinants to Health [Internet]. Project HOPE; 2014 [cited 2020 Jul 26]. Available from: https://www.healtha ffairs.org/do/10.1377/hpb20140821.404487/full/

\section{Publisher's Note}

Springer Nature remains neutral with regard to jurisdictional claims in published maps and institutional affiliations.

Ready to submit your research? Choose BMC and benefit from:

- fast, convenient online submission

- thorough peer review by experienced researchers in your field

- rapid publication on acceptance

- support for research data, including large and complex data types

- gold Open Access which fosters wider collaboration and increased citations

- maximum visibility for your research: over $100 \mathrm{M}$ website views per year

At BMC, research is always in progress.

Learn more biomedcentral.com/submission 